\title{
Effect of Embedded Sensory Stimulation in the Design of Learning Framework for Increasing Attention Span of Children with Autism
}

\author{
Bilikis 0 Banire* \\ Department of Information and Communication Technology, Hamad Bin Khalifa University, Qatar
}

Submission: April 27, 2017; Published: May 26, 2017

*Corresponding author: Dena Al Thani, Marwa Qaraqe, Department of Information and Communication Technology, Hamad Bin Khalifa University, KSA, Email: bbanire@hbku.edu.qa; dalthani@hbku.edu.qa; mqaraqe@hbku.edu.qa

\section{Abstract}

The inattention deficit in children with autism has made teaching a challenge for teachers, parents and caregivers. This inattention also affects the triad of impairment: social, communication and pattern of behavior associated with them. Education has been identified as a relative cure for their impairments and use of technology as a medium for learning has been said by different studies as an effective way of teaching children with autism. Hence, some studies have come up with learning frameworks for developing educative software applications which focus on gaining the attention of the children with a learning disability such as autism during learning. Thus, this review presents existing learning frameworks in developing effective software learning system for children with autism.

Keywords: Autism; Learning framework; Sensory stimulations

\section{Introduction}

Children with Autism Spectrum Disorders are characterized with stereotypical behaviours, communication and social impairment [1]. However, the degree these behaviours vary from one child to another [2]. Other deficits that can be found in them are inattention in learning, low cognitive skills such that they fail to comprehend expression in the actual context except it is represented in pictures [3]. They lack coherence in constructing ideas together as a whole and cannot maintain on a topic. Due to these deficits, majority of them require life-long support from their caregivers such as parents, teacher and other people who interact with them. Sometimes, these caregivers get overwhelmed with the demands of these children. However, some level of training can assist these children to be relatively self-dependent as some of them possess some genius attributes which varies from one person to another. For example, some can memorize several texts at a glance (hyperlexia), understanding visual beyond the reasoning of a typical person, dealing with number calculations and others [4]. Some of these traits were found in a renowned professor with autism; "Mary Temple Gradin" who studied Animal Science at Colorado State University. This professor would not have achieved that level of academic excellence if nothing was done to intervene the known deficits of autism in her. These deficits were managed with several therapies and interventions.

Hence, interventions are required in maximizing the potentials of these children to benefit them, their families, and the society at large. Having read from Center for Diseases Control and Prevention (CDC 2016) [5] that the population of people with autism has been on the increase and their ratio to typical children had increased from 1 in 150 children to 1 in 68 between year 2000 and 2012, then this brings about the need for more technological interventions to augment the existing ones which can go a long way in making them relatively self-dependent.

\section{Autism and Attention in Learning}

Inattention in children with autism occurs when the irrelevant stimulus is attended to rather than the relevant stimuli [6]. This simply explains attending to another thing other than the learning content. The retention of knowledge can only be achieved when attention is paid to the medium of learning. Attention is then considered as a fundamental factor that facilitates teaching [7]. The degree of inattention in learning task found in children with autism is higher as compared to typical children and what captures their attention also differs. Therefore, educating them 
requires a lot more effort from teachers as compared to typical children [8]. Some software learning application systems have been developed to support their attention during learning using simple pictorial illustrations (such as humanoid and real world object) to teach different skills like social skills, and pet recognition [9-11].

Another factor of inattention in children with ASD is the difficulty in handling sensory stimulations and reactions in the learning environment [12]. These stimulations make them exhibit self-stimulating behaviours to help them balance out the unlimited or limited sensory input depending on the child (such self-stimulating behaviours could be restlessness, inflicting selfinjuries, inattention, and others) [8]. Hence, sensory room serve as therapy for providing these children with required stimulation which provides them with calming effects and make them ready to learn. There are different categories of attention in children with autism and they include:

\section{Shifting attention}

When learners orientate their focus from one object to another.

\section{Focus of attention}

This is seen in a situation where the learners focus their attention on small portion or large portion of a learning interface. For example, when a child with autism wants a chocolate they look at chocolate, try to advance towards it and make eye contact with the person offering the chocolate. Some try to mumble in to demand for the chocolate while the less verbal children continue to point at the chocolate.

\section{Attentional cueing}

This is the idea of directing the attention of learner to an item that requires his or her attention with a cue or guide (such as arrow or highlighting with colors).

\section{Social attention}

This is when learners give attention to social item in a learning environment [13].

\section{Theorie and Strategies of Attention}

Attention involves attending to a task with the influence of motivating factors be it internal or external motivation [14]. In other words, the strategies of attention involve the need for a learner to perform task that motivates or interest him during learning. More so the manner of attending to task varies from one person to another based on their cognitive skills. Motivation as a strategy of attention considers relevance, confidence, and satisfaction $[15,16]$. Other factors of strategies for attention in learning are defined below:

\section{Humour}

Providing learners with humour such as visuals that may interest the learner can contribute to more attention span.

\section{Variability}

This involves the use of several media (such as video, images, animations and others) The use of colourful images, animated characters and sound can increase the attention of children with autism in teaching social skills as they find this type of visuals attractive and interesting to them [10].

\section{Correctness}

This involves the use of right visuals for specific examples.

\section{Concreteness}

Providing leaners with concrete examples and give them the opportunity of paying more attention to the learning content.

\section{Inquiry}

This involves brain storming such as giving questions to the learner to answer.

\section{Participation}

This involves engaging the student in the learning activity such as hands on, role play of the learning content. This might not be applicable to some children with autism because of the impairment in motor skills and attending to multiples of instructions at a time.

\section{Incongruity}

This involves the presentation of idea in an unusual way to catch the attention of the learner.

\section{Existing Framework for Developing Learning System for Children with Autism}

Educating children with autism with the skills they lack at an early stage are of more benefit than at adult stage. However, some interventions must have failed with some individual with autism and yet worked for others because of the variation in impairments found in them [17]. More so, the learning style of these children varies so it is imperative to investigate the style of learning and their interest before the development of a learning system. There are several frameworks designed for developing learning systems for children with autism and they support the attention span of these children. Some of these frameworks are discussed below.

\section{Reach and teach framework}

Reach and teach framework was designed to enhance caregivers in teaching social skills of children with autism who have difficulties in responding to social interactions in a way and manner that will capture and increase their attention towards the learning objective [10]. This framework has used representational illustrations such as video, animations, text, audio, and images. The learning objective was to improve the impairment in their "Theory of Mind". Children with autism are affected with the impairment in generalizing things such that they cannot comprehend indirect expression $[18,19]$. 
The purpose of these components in this framework is to capture the attention of children with autism by using different style of presentations due to their differences in learning style. Different animation games were incorporated to motivate the children's interest in learning. The game has a set time so that it restricts the child from diverting from the main content of learning. The study of this framework found out that many of the children (90 percent) preferred the animation mode of presentation, 40 percent were inclined towards image mode of presentation, 10 percent preferred video mode of presentation and none was interested in to audio and text mode.

\section{Learning framework for children with autism (LeFCA)}

The purpose of the LeFCA is to guide the development of a learning system to teach children with autism using four different types of games to enhance their ability of matching, identifying and labeling objects and these features aid long-term learning [11]. The main technique here was visual learning and not audio. This contrast the Reach and Teach framework. Another technique they used in sustaining the attention these children was to give a feedback on their performance on the game. The feedbacks were of two forms: positive feedback to commend and encourage the child to continue playing and the other was correction feedback to guide the child for any mistake in identifying the shapes. The result of the study showed that children with less severe autism could learn the shapes while the children with severe autism took more time in recognizing the shapes.

\section{Visual hybrid development learning system (VHDLS) framework}

VHDLS framework was based on two different types of visuals: Representational and Abstract as mode of learning [20]. This framework has based these two types of visuals on methods used in Picture Exchange Communication System (PECS) and visual sensory room activity respectively. The representational is used to illustrate the content of what is to be learnt using pictures, video, and humanoid cartoon while the abstract visual was used to provide the children with visual stimulation with animation different colours as used in sensory room. The result of the study showed using both the representational and abstract visuals together increased the attention span of children with autism as compared to using representational alone.

\section{Conclusion}

The findings from this review have shown that children with autism have inattention amongst other impairments such as social and communication impairments. The inattention in learning is may be due to inadequate sensory stimulation they get from the learning environment. Hence, teaching these children has been challenging for teachers. Thus, studies have developed software learning system to enhance the attention of children with learning disability such as autism. Some of the learning frameworks used in the design and development of these learning tools that captures the attention of these children are Reach and Teach framework, LeFCA framework, and VHDLS framework.

These frameworks considered visual and audio style of learning such as animations, video and colourful images but other sensory stimulations such as proprioceptive and vestibular during learning were not included and these may contribute to better attention span of children with autism. Hence, instead of limiting the sensory stimulation to multimedia alone, other sensory stimulation should be leveraged.

\section{Reference}

1. Kanner L (1973) Childhood psychosis: Initial studies and new insights. VH Winston \& Sons, USA.

2. Wing, L, Gould J (1979) Severe impairments of social interaction and associated abnormalities in children: Epidemiology and classification. Journal of autism and developmental disorders 9(1): 11-29.

3. Grandin T (2006) Thinking in pictures: And other reports from my life with autism. Vintage, UK.

4. Wetherby AM, Prizant BM (2000) Autism spectrum disorders: A transactional developmental perspective. Brookes Pub, USA.

5. (2017) Prevention, C.f.D.C.a. Autism Spectrum Disorder (ASD).

6. Pashler HE, Sutherland S (1988) The psychology of attention. MIT press, England.

7. Moore M, Calvert C (2000) Brief report: Vocabulary acquisition for children with autism: Teacher or computer instruction. J Autism Dev Disord 30(4): 359-362.

8. Roberts JE, King-Thomas L, Boccia ML (2007) Behavioral indexes of the efficacy of sensory integration therapy. Am J Occup Ther 61(5): 555562.

9. Konstantinidis EL, Luneski A, Frantzidis CA, Nikolaidou M, HitoglouAntoniadou M, et al. (2009) Information and communication technologies (ICT) for enhanced education of children with autism spectrum disorders. The Journal on Information Technology in Healthcare 7(5): 284-292.

10. Doyle T, Arnedillo-Sánchez I (2011) Using multimedia to reveal the hidden code of everyday behaviour to children with autistic spectrum disorders (ASDs). Computers \& Education 56(2): 357-369.

11. Hulusic V, Pistoljevic N (2012) "LeFCA": Learning Framework for Children with Autism. Procedia Computer Science 15: 4-16.

12. Tomchek SD, Dunn W (2007) Sensory processing in children with and without autism: a comparative study using the short sensory profile. Am J Occup Ther 61(2): 190-200.

13. Ames C, Fletcher-Watson S (2010) A review of methods in the study of attention in autism. Developmental Review 30(1): 52-73.

14. Hale G (2012) Attention and cognitive development. Springer Science \& Business Media, Germany.

15. Walsh L (2009) The design of multimedia based instruction to support the academic and social development of children with autism, Waterford Institute of Technology, Ireland.

16. Keller JM (1987) Development and use of the ARCS model of instructional design. Journal of instructional development 10(3): 2-10.

17. Case-Smith J, Bryan T (1999) The effects of occupational therapy with sensory integration emphasis on preschool-age children with autism. American Journal of Occupational Therapy 53(5): 489-497. 
18. Baron-Cohen S (1995) Mindblindness: An essay on autism and theory of mind. The MIT Press, England.

19. Baron-Cohen S (2000) Theory of mind and autism: A review. International review of research in mental retardation 23: 169-184.
20. Banire B, Jomhari N, Ahmad R (2015) Visual Hybrid Development Learning System (VHDLS) Framework for Children with Autism. J Autism Dev Disord 45(10): 3069-3084.

\section{Your next submission with Juniper Publishers will reach you the below assets}

- Quality Editorial service

- Swift Peer Review

- Reprints availability

- E-prints Service

- Manuscript Podcast for convenient understanding

- Global attainment for your research

- Manuscript accessibility in different formats

( Pdf, E-pub, Full Text, Audio)

- Unceasing customer service

Track the below URL for one-step submission https://juniperpublishers.com/online-submission.php 